\title{
Fibre DFB lasers in a 4x10 Gbit/s WDM link with a single sinc-sampled fibre grating dispersion compensator
}

\author{
Morten Ibsen, Alexander Fu, Harald Geiger and Richard I. Laming
}

\begin{abstract}
:
WDM transmission and dispersion compensation at $40 \mathrm{Gbit} / \mathrm{s}$ over $200 \mathrm{~km}$ standard fibre is demonstrated on a $100 \mathrm{GHz}$ grid using four high power single-polarisation single-sided output DFB fibre laser based transmitters and a single 4 channel WDM chirped fibre Bragg grating dispersion compensator.
\end{abstract}

\section{Introduction}

WDM transmission systems have received growing attention over the last few years because of their obvious advantages when upgrading existing systems to operate at higher data-rates. Operating at bit-rates of $10 \mathrm{Gbit} / \mathrm{s}$ and above requires compensation of the chromatic dispersion suffered in the existing fibres. Dispersion compensation with single channel chirped fibre gratings has been demonstrated on numerous occasions [1,2]. Transmitting multiple WDM-channels the relative channel spacings are of great importance. The periodic spectral response and identical multiple channel characteristics of the recently developed sinc-sampled grating [3] makes it a very attractive WDM device.

DFB fibre lasers exhibit ideal source characteristics being an inherently fibre compatible device. The operating wavelengths [4] can readily be manufactured with high accuracy to achieve a constant frequency separation using current grating writing techniques [5]. They can also be designed to lase in a single polarisation whilst single-sided

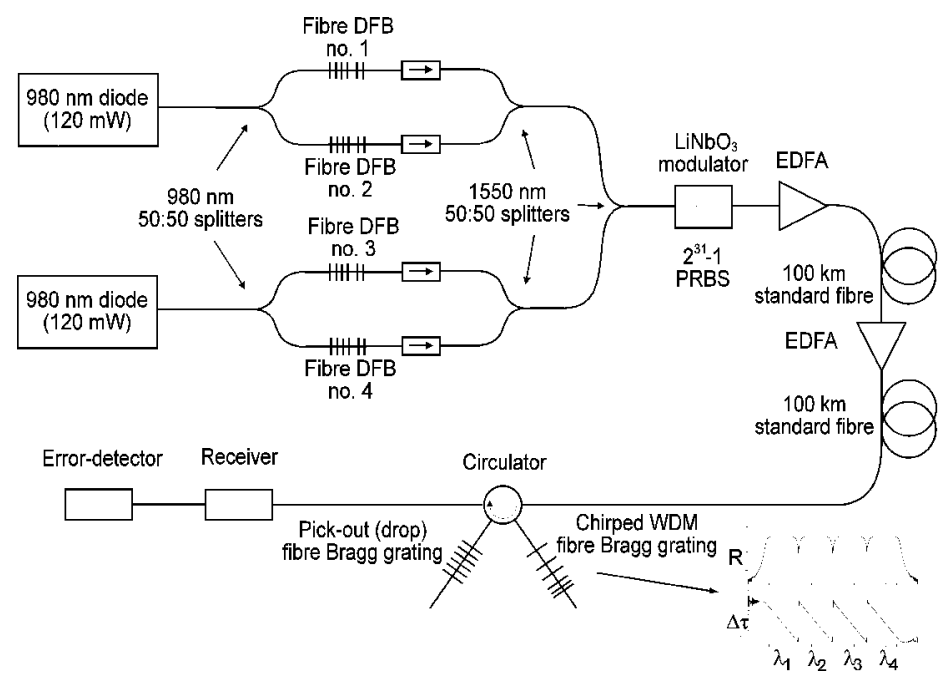

Fig. 1: Schematic diagram of the experimental setup. lasing output by offsetting the phase-shift from the centre position [6] increases the potential further.

In this paper we combine the devices and demonstrate for the first time a WDM system comprising 4 high power DFB fibre lasers operating with single sided and single polarisation outputs. The lasers are demonstrated to be reliable signal sources in a $4 \times 10 \mathrm{Gbit} / \mathrm{s}, 100 \mathrm{GHz}$ channel specing WDM transmission experiment over 200 $\mathrm{km}$ of standard fibre with robust dispersion compensation performed by a single 4 channel 100 $\mathrm{GHz}$ spacing chirped sinc-sampled fibre Bragg grating.

\section{Experiment}

The experimental setup (Fig. 1) consists of 4 single polarisation asymmetric DFB-fibre lasers of length $5 \mathrm{~cm}$ separated in frequency by $100 \mathrm{GHz}$. The lasers have $\lambda / 4$ phaseshifts offcentred by $4 \mathrm{~mm}$. Each is pumped at $980 \mathrm{~nm}$ with $60 \mathrm{~mW}$ power from a pump diode, resulting in single sided output powers of $7.1 \mathrm{dBm}, 7.7 \mathrm{dBm}, 7.0 \mathrm{dBm}$, and 7.7 $\mathrm{dBm}$, and operating wavelengths of $1547.6 \mathrm{~nm}$, $1548.4 \mathrm{~nm}, 1549.2 \mathrm{~nm}$ and $1550.0 \mathrm{~nm}$ respectively. The signal to noise ratio between the 4 DFB signal channels is in excess of $50 \mathrm{~dB} \quad(0.08 \mathrm{~nm}$ resolution) and the linewidth of the devices has been measured to be $\sim 10$ $\mathrm{kHz}$.

The polarisation states of the lasers are individually aligned using polarisation controllers to allow modulation in a single modulator. The excess power of the lasers allows their output to be combined in an all-fibre multiplexer consisting of three $1550 \mathrm{~nm} 3 \mathrm{~dB}$ splitters, before each channel is modulated using a lithium niobate $\left(\mathrm{LiNbO}_{3}\right)$ modulator. Fig. 2a shows the output spectra of the 4 DFB fibre 
lasers measured directly after the modulator. The WDM channels are amplified and then transmitted over a $200 \mathrm{~km}$ standard telecommunication fibre $(\mathrm{D}=16 \mathrm{ps} / \mathrm{nm} / \mathrm{km} @ 1.55 \mu \mathrm{m})$ link. Ultimately the high power of the fibre DFBs will obviate the requirements for amplification prior to transmission. After transmission the channels are simultaneuosly dispersion compensated with a 4 channel sinc-sampled fibre grating [3], which is wavelength matched to the transmitted channels (Fig. 2). The grating has a sampling period of 1 $\mathrm{mm}$ and is $25.8 \mathrm{~cm}$ long. It is chirped over $0.8 \mathrm{~nm}$ and is apodised over $10 \%$ of the total length at either end to reduce the ripples in the dispersion characteristics. Each dispersion-compensating channel of the grating exhibit a reflectivity of $\sim 60$ $\%$ and dispersions of $-3263 \mathrm{ps} / \mathrm{nm},-3206 \mathrm{ps} / \mathrm{nm}$, $-3250 \mathrm{ps} / \mathrm{nm}$ and $-3238 \mathrm{ps} / \mathrm{nm}$ respectively with a maximum in-band time delay ripple of $\sim 50 \mathrm{ps}$ (Fig. $2 b \& c)$. A uniform fibre grating used in an adddrop configuration, selects the channel under test and drops it onto the receiver and error detector. All the fibre gratings used in this experiment are manufactured using a continuous grating writing technique [3] which allows very complex grating structures to be formed.

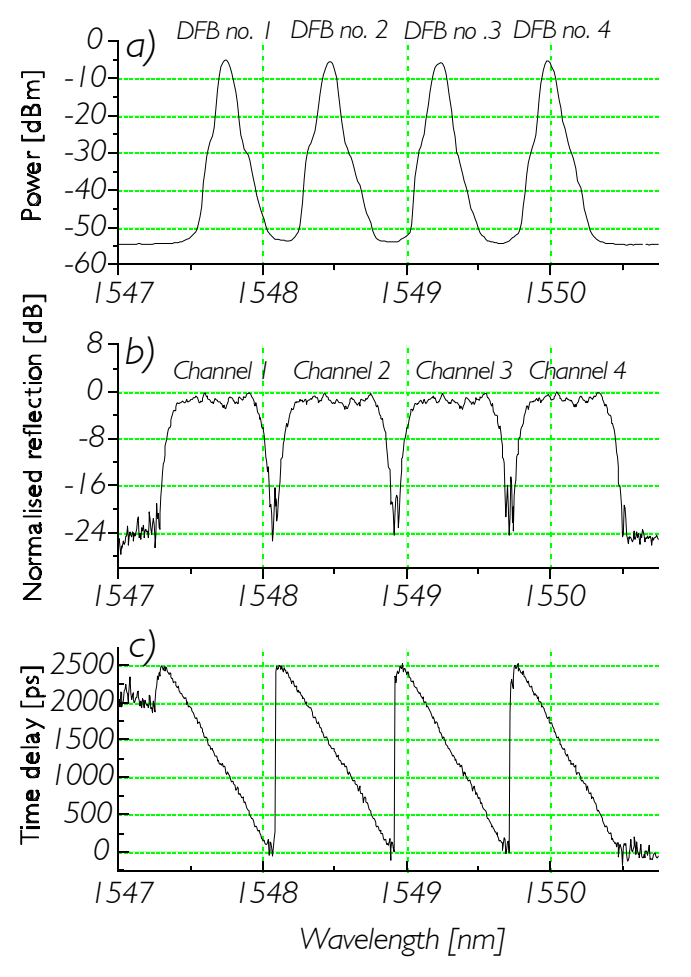

Fig. 2a) DFB lasing spectra after the modulator. 2b) Reflection and c) time delay characteristic of the 4 channel chirped sinc sampled fibre Bragg grating.

\section{Results and discussion}

Bit error rate (BER) vs. received power for the 4 dispersion compensated channels and back-to back with DFB laser no.1 are shown in Fig. 3. The figure shows a maximum power penalty of $1 \mathrm{~dB}$ for error free operation. This variation could be due

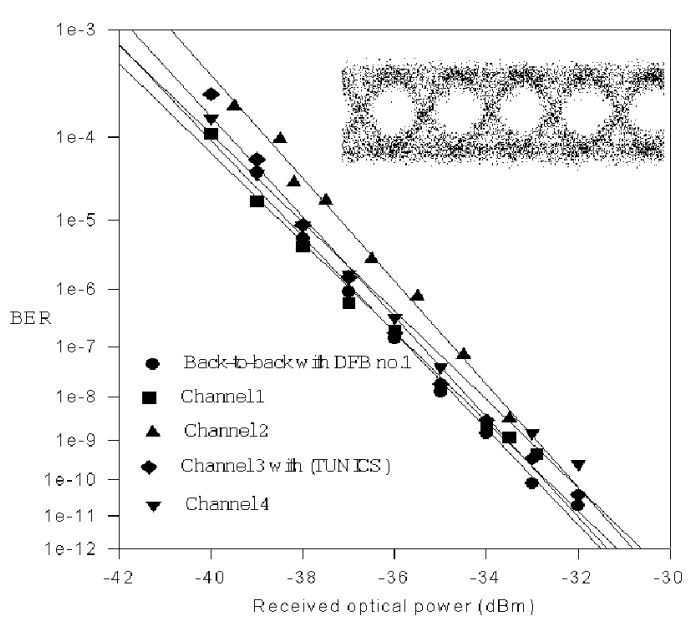

Fig. 3: Bit error rate performance of simultaneous 4x10Gbits WDM transmission system. (Insert) Dispersion compensated eye of channel 4 .

to the fact that a different pick-out grating is used for each channel. In order to test the quality of the dispersion compensating grating, a wavelength scan across the bandwidth of channel 2 was made, but not shown here due to limited space. In this case the received power was kept constant at -34.5 $\mathrm{dBm}$ and the BER was recorded.

A variation of 2 orders of magnitude in the error rate around $10^{-8}$ was measured, corresponding to a less than $\pm 0.75 \mathrm{~dB}$ power penalty (Fig. 3 ). The effect of cross talk between adjacent channels was also evaluated by comparing BER data for the WDM system with data obtained when transmitting only one of the wavelength channels at a time. No degradation in the performance of the system was observed. In order to compare the performance of the fibre DFB laser transmitters with a traditionally used tunable laser source (TUNICS) a single channel BER measurement tuned onto channel 3 using this source was performed. Again no significant difference was observed (Fig. 3). 


\section{Conclusions}

We have demonstrated the first 4 channel 40 Gbit/s NRZ WDM transmission experiment over a $200 \mathrm{~km}$ standard fibre link employing high power all-fibre DFB lasers as signal sources. Simultaneous dispersion compensation of the 4 channels was performed using a sinc-sampled multichannel fibre grating that exhibits identical dispersion characteristics on a $100 \mathrm{GHz}$ comb of wavelength channels. We believe that this experiment demonstrates that the technology of fibre DFB lasers has matured and that they are an attractive transmitter alternative to semiconductor DFB lasers and are ready to take part in the realisation of all-fibre WDM transmission systems.

\section{Acknowledgements}

The authors wish to thank Martin J. Cole and Michael K. Durkin for help in establishing the grating writing setup, Erland Rønnekliev and Gregory J. Cowle for discussions on DFB fibre lasers, together with Sze Y. Set and Perikles Petropoulos for system oriented aspects. This work was in part supported by Pirelli Cavi SpA, Milan. The Optoelectronics Research Centre is an EPSRC (UK) funded interdisciplinary research centre.

\section{References}

1. Ouellette, F., Krug, P.A., Stephens, T., Dhosi, G. and Eggleton, B.J.: 'WDM and broadband dispersion compensation using chirped sampled fibre Bragg gratings', Electron. Lett., 31, (11), pp. 899-901, 1995.

2. Dong, L., Cole, M.J., Ellis, A.D., Durkin, M., Ibsen, M., Gusmeroli, V. and Laming, R.I.: ' $40 \mathrm{Gbit} / \mathrm{s} 1.55 \mu \mathrm{m}$ transmission over $109 \mathrm{~km}$ of nondispersion shifted fiber with long continuously chirped fiber gratings', OFC 97, paper PD6, Dallas, USA.

3. Ibsen, M., Durkin, M.K., Cole, M.J. and Laming, R.I.: 'Sinc-sampled fibre Bragg gratings for identical multiple wavelength operation', IEEE Photon. Technol. Lett., 10, (6), pp. 842-844, 1998.
4. Hhbner, J., Varming, P. and Kristensen, M.: 'Five wavelength DFB fibre laser source for WDM systems', Electron. Lett., 33, (2), pp. 139-140, 1997.

5. Cole, M.J., Loh, W.H., Laming, R.I., Zervas, M.N. and Barcelos, S.: 'Moving fiber/phase mask scanning technique for enhanced flexibility in producing fiber gratings with a uniform phase mask', Electron. Lett., 31, (12), pp. 1488-1489, 1995.

6. Lauridsen, V.C, Srndergaard, T., Varming, P. and Povlsen, J.H.: 'Design of distributed feedback fibre lasers', ECOC 97, 3, Session WeC1, pp. 39-42, Edinburgh, UK. 